\title{
Valley dependent superconducting proximity effect in a twisted van der Waals heterojunction
}

\author{
Jing-Jing Xian, ${ }^{1,}{ }^{*}$ Li Chen,,${ }^{1, *}$ Xin Liu, ${ }^{1}$ Wen-Hao Zhang $\odot,{ }^{1}$ Lang Peng, ${ }^{1}$ Rui Li,${ }^{1}$ Min Cai, ${ }^{1}$ \\ Jingsi Qiao, ${ }^{2}$ and Ying-Shuang Fu ${ }^{1, \dagger}$ \\ ${ }^{1}$ School of Physics and Wuhan National High Magnetic Field Center, Huazhong University of Science and Technology, \\ Wuhan 430074, China \\ ${ }^{2}$ Centre for Advanced 2D Materials and Graphene Research Centre, National University of Singapore, Singapore 117546, Singapore
}

(Received 9 June 2020; revised 14 August 2020; accepted 17 August 2020; published 3 September 2020)

\begin{abstract}
Leakage of Cooper pairs through heterointerfaces leads to the superconducting proximity effect, which has been utilized extensively in building functional quantum devices and inducing novel superconductivity. While an atomically sharp interface in real-space is known to be crucial for effective Cooper pair proximity transfer, the influence of electronic valleys in the momentum space has not been sufficiently investigated. Here, we report the observation of valley dependent superconducting proximity effect in a heterostructure with twisted overlapping. The heterostructure is realized by growth of multidomain $\mathrm{Bi}(111)$ films on a single-crystal $\mathrm{NbSe}_{2}$ substrate with molecular beam epitaxy. With spectroscopic imaging scanning tunneling spectroscopy, we identified different types of atomic overlapping in the Bi films, and measured drastic changes of proximity-induced superconducting gap sizes on the differently oriented Bi domains. Based on our theoretical model calculation, this phenomenon can be interpreted as valley dependent superconducting proximity coupling between the Bi film and $\mathrm{NbSe}_{2}$. We also investigated the lateral proximity effect between two adjacent Bi domains, which determines a significant reduction of the mean free path of electrons, associated with interfacial scattering. Our study expands the scope of tunable physical properties with the valley degree of freedom.
\end{abstract}

DOI: 10.1103/PhysRevResearch.2.033360

\section{INTRODUCTION}

The electron valleys, local extrema in band structures, have long been known in three-dimensional (3D) bulk materials such as bismuth and $\mathrm{Si}$ [1-3]. Their existence in two-dimensional (2D) materials, such as graphene and transition metal dichalcogenides, is feasible for control via external fields and has revived immense interest [4,5]. Electron valleys not only serve as an alternative degree of freedom for information carriers in additional to charge and spin, but also have been exploited for engineering the physical properties of quantum systems. In inversion asymmetric systems, the electron valleys function as pseudospins on the electron motion, coming from their contrasting Berry curvatures at momentum space [6], resulting in novel transport of the valley Hall effect [7], dichroism of circularly polarized lights [8,9], and Isingtype superconducting pairing [10].

The superconducting proximity effect, on the other hand, is a fundamental phenomenon that occurs at superconductor/normal metal or superconductor/superconductor interfaces, exhibiting leakage of Cooper pairs through an Andreev

\footnotetext{
*These authors contributed equally to this work. ${ }^{\dagger} \mathrm{yfu} @$ hust.edu.cn
}

Published by the American Physical Society under the terms of the Creative Commons Attribution 4.0 International license. Further distribution of this work must maintain attribution to the author(s) and the published article's title, journal citation, and DOI. process [11]. The superconducting proximity effect has been utilized to achieve unconventional triplet pairing in a ferromagnetic metal [12], effective $p$-wave pairing for topological superconductivity in semiconductor nanowires [13], ferromagnetic atomic chains [14], and topological surface states $[15,16]$, as well as to build functional quantum devices $[17,18]$. The transmission of the superconducting proximity effect relies sensitively on the overlapping integral of wave functions at the interface [19,20], and the momenta coupling [16]. Thus, if the band structure of the superconductor and the normal metal are endowed with valleys, their superconducting proximity effect will be dependent on the moiré overlapping. Such valley mediated superconducting pairing is intriguing, which, however, has not been sufficiently studied experimentally.

Here, we report the observation of valley dependent superconducting proximity effect in a heterostructure realized by growth of $\mathrm{Bi}$ films on a $\mathrm{NbSe}_{2}$ substrate with molecular beam epitaxy. With scanning tunneling microscopy and spectroscopy (STM/STS), we identified different twisting angles in the heterostructure and measured drastic changes in the superconducting gap sizes on $\mathrm{Bi}$ films of different domains from the proximity effect. This phenomenon is interpreted as Andreev reflection which depends on valley coupling in the in-plane momentum space with our theoretical modeling.

\section{METHODS}

The experiments were performed in a custom made Unisoku STM (1300) system. High-purity Bi (Alfa Aesar, 
(a)

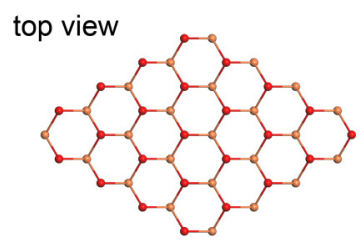

side view

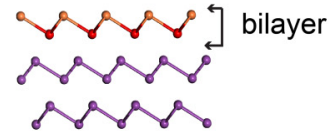

(c)

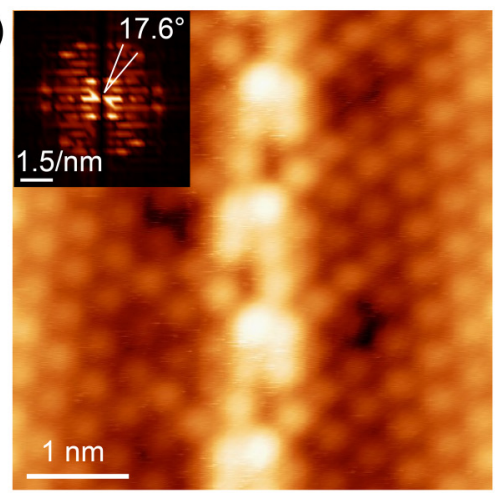

(b)
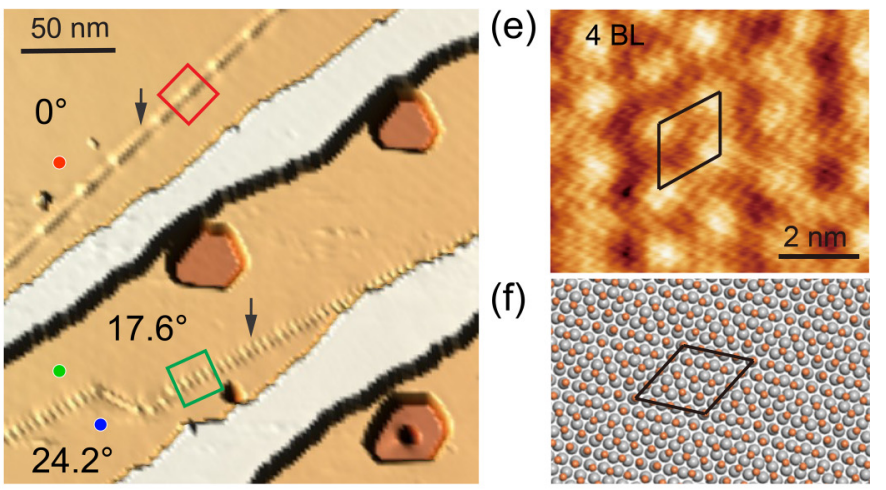

(f)

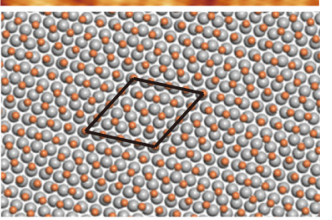

(d)
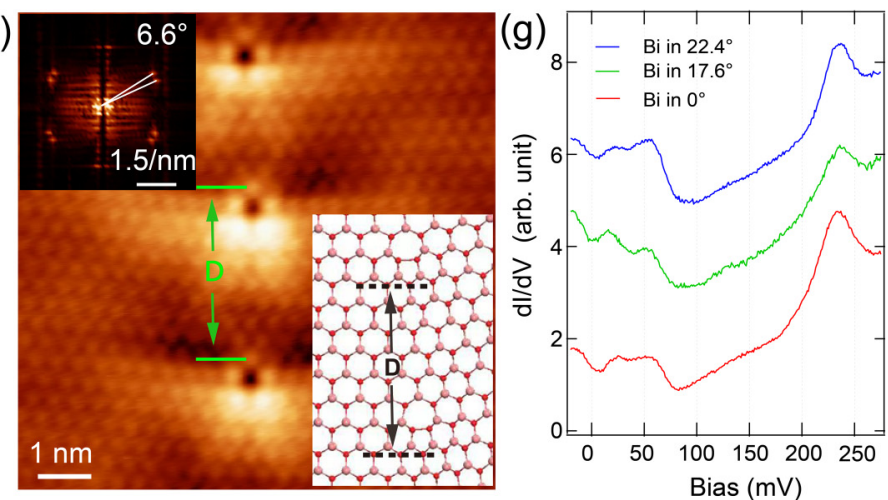

FIG. 1. Morphology and electronic structure of $\mathrm{Bi}(111)$ films on $\mathrm{NbSe}_{2}$ substrate with different twisting angles. (a) Top (up) and side view (down) of crystal structure of $\mathrm{Bi}(111)$ film. (b) Pseudo-3D STM image $\left(V_{\mathrm{b}}=1 \mathrm{~V}, I_{t}=10 \mathrm{pA}\right)$ of $\mathrm{Bi}(111)$ films with dominating thickness of 8 $\mathrm{BL}$. The twisting angles between $\mathrm{Bi}$ and $\mathrm{NbSe}_{2}$ are indicated. Two black arrows mark the domain boundaries. (c), (d) Zoomed-in STM images of two domain boundaries with atomic resolution. The images in (c) $\left(V_{\mathrm{b}}=-100 \mathrm{mV}, I_{\mathrm{t}}=500 \mathrm{pA}\right)$ and $(\mathrm{d})\left(V_{\mathrm{b}}=4 \mathrm{mV}, I_{t}=500 \mathrm{pA}\right)$ are obtained at the area marked with red and green boxes in (b), respectively. Inset images in (c), (d) are their respective fast Fourier transformations. Structural model of the defects with interspacing $D$ in the domain boundary of (d) is also shown in its inset. The pink and red balls represent top and bottom Bi atoms in a single double layer, respectively. (e) STM image $\left(V_{\mathrm{b}}=400 \mathrm{mV}, I_{\mathrm{t}}=100 \mathrm{pA}\right)$ of a 4-BL Bi(111) film on $\mathrm{NbSe}_{2}$ substrate, showing moiré patterns. (f) Ball model showing the atomic stacking at the $\mathrm{Bi} / \mathrm{NbSe}_{2}$ interface. The gray and orange balls represent Se and Bi atoms, respectively. The black rhombuses in (e), (f) mark the moiré unit. (g) Tunneling spectra $\left(V_{\mathrm{b}}=300 \mathrm{mV}, I_{\mathrm{t}}=100 \mathrm{pA}\right.$, $V_{\mathrm{mod}}=14.14 \mathrm{mV}$ ) of Bi films of (b) with different twisting angles relative to $\mathrm{NbSe}_{2}$.

99.999\%) atoms were evaporated to the in situ cleaved $\mathrm{NbSe}_{2}$ substrate at room temperature, followed by postannealing at $520 \mathrm{~K}$ for $10 \mathrm{~h}$ to form uniform films [21-23]. The STM measurements were performed at an effective temperature of $2.2 \mathrm{~K}$ with a $\mathrm{W}$ tip. The tunneling spectra were obtained by lock-in detection of the tunneling current at $983 \mathrm{~Hz}$.

\section{RESULTS}

Bulk Bi has a rhombohedral crystal structure, which can be considered as a stack of weakly bonded Bi bilayers (s) with $A B C$ type stacking along its [111] direction [Fig. 1(a)]. Within each BL, the Bi atoms are covalently bonded forming a buckled honeycomb structure with a height of $0.41 \mathrm{~nm}$. Only the top $\mathrm{Bi}$ atoms of the $\mathrm{BL}$ can be imaged, giving rise to a triangular lattice in STM images. Such Bi (111) films can be grown on $\mathrm{NbSe}_{2}$ substrates in a layer-by-layer mode via van der Waals epitaxy [21,22]. Figure 1(b) shows the topography of as-grown Bi film whose thickness is dominated by $8 \mathrm{BL}$. There are two stripe shaped islands and three hexagonal pits whose heights are all single BLs. Notably, two domain boundaries (marked with black arrows) run straight across the wide terrace, dividing the 8-BL film into three domains.
Zoomed-in images of the domain boundaries reveal the domain orientations and the atomic structure of the boundaries. Figures 1(c) and 1(d) are high-resolution images taken at the selected areas of the two domain boundaries of Fig. 1(b). The real-space atomic-resolution images, in conjunction with their fast Fourier transformations, indicate the relative tilting angles between the two neighboring domains are $\phi=17.6^{\circ}$ in Fig. 1(c) and $\phi=6.6^{\circ}$ in Fig. 1(d), respectively. The $17.6^{\circ}$ domain boundary has a periodic protrusion of $2.58 \mathrm{~nm}$ [Fig. 1(c)], which may presumably originate from straininduced local lattice distortion that accumulates when the two domains meet with a large tilting angle. The superimposed regular lattices of the two domains indicate the lateral extension of the modulation happens within a unit cell. The $6.6^{\circ}$ domain boundary, on the other hand, is composed of periodic dark spot defects with a spacing of $3.6 \mathrm{~nm}$ [Fig. 1(d)]. There are five bright atoms surrounding the dark spot, which nicely fits to a $5 \mid 7$ core structural model in the inset of Fig. 1(d). In the Burgers model, the distance $D$ between the adjacent dislocation cores is $D \approx\left(n+\frac{1}{2}\right) \sqrt{3} a$, where $a=0.45 \mathrm{~nm}$ is the in-plane lattice constant of $\mathrm{Bi}$, and $n$ is the integer number of Bi columns between adjacent dislocation cores [24]. When the misorientation angle $\theta$ is small, $\theta \approx \frac{1}{\sqrt{3(n+1 / 2)}}$. Therefore, 
a tilting angle $\theta=6.6^{\circ}$ results in $n=8$ and $D \approx 3.5 \mathrm{~nm}$, which is consistent with our observation.

The lattice orientation of the $\mathrm{NbSe}_{2}$ substrate can be determined by the moire patterns observed on a thin 4-BL film [Figs. 1(e) and 1(f)], and further confirmed by imaging the clean $\mathrm{NbSe}_{2}$ substrate prior to the growth of $\mathrm{Bi}$. Thus, the stacking angles of the three $\mathrm{Bi}$ domains in Fig. 1(b) are thereafter designated as $0^{\circ}, 17.6^{\circ}$, and $24.2^{\circ}$, respectively. Subsequently, we characterize the electronic properties of the domains and the domain boundaries. Tunneling spectra measured at different domains show identical spectroscopic features at large energy ranges, demonstrating their electronic properties are not affected by the stacking angle relative to the substrate [Fig. 1(g)]. They all feature a strong peak at $235 \mathrm{meV}$, which arises from the van Hove singularity of the Bi films. This peak, as indicated in our previous study [21], shifts monotonically to lower energy with increasing thickness of the $\operatorname{Bi}(111)$ film, due to the evolution of band structure with thickness [25]. Thus, this peak, acting as a hallmark, allows the spectroscopic determination of the film thickness.

Next, we investigate the superconducting proximity effect induced on the Bi films from the $\mathrm{NbSe}_{2}$ substrate. Normalized tunneling spectra acquired at interior regions of each domain all show prominent superconducting gaps [Fig. 2(a)], which intriguingly have different gap sizes and different intensity of coherence peaks. We fit the superconducting gap with the function $\frac{d I}{d V} \propto \int_{-\infty}^{+\infty} \operatorname{Re}\left[\frac{\left|E-i \Gamma_{D}\right|}{\sqrt{\left(E-i \Gamma_{D}\right)^{2}-\Delta^{2}}}\right] \frac{\partial f(E+e V, T)}{\partial E} d E$, where $f(E+e V, T)$ is the Fermi-Dirac distribution, $T$ is the effective temperature, $\Gamma_{D}$ is quasiparticle damping in the Dynes equation, and $\Delta$ is the superconducting gap size. The fitting [Fig. 2(b)] delivers gas sizes of $0.52,0.67$, and $0.69 \mathrm{meV}$ for the domains of $0^{\circ}, 17.6^{\circ}$, and $24.2^{\circ}$, respectively [Fig. 2(c)]. Concomitantly, the normalized zero-bias conductance (ZBC) of the superconducting gap is also highest for the $0^{\circ}$ domain, and barely distinguishable for the $17.6^{\circ}$ and $24.2^{\circ}$ domains [Fig. 2(c)]. The effective temperature given by the fitting is $2.2 \mathrm{~K}$. The superconducting gaps are uniform within the interior of the domains except at the domain boundary (Fig. 3). Since the two domains are endowed with different sizes of the superconducting gaps, they form a lateral junction of two superconductors, S1/S2, and thus the lateral proximity effect gets involved, whose details will be formulated later.

The variation of superconducting gaps on different Bi domains signifies the contribution of the interfacial stacking angle relative to the $\mathrm{NbSe}_{2}$ substrate on the proximity effect. We thus turn to investigate the superconducting proximity effect of thinner Bi films of 4 BL [Fig. 4(a)]. In this case, the twisted overlapping at the interface is closer to the film's surface, expecting more enhanced influence on the angle dependent superconducting proximity effect. Figures 4(b) and 4(c) show tunneling spectra measured at the interior and the boundary of two domains of $0^{\circ}$ and $24.2^{\circ}$. As expected, their superconducting gaps are larger than that of the 8-BL films, due to the shorter distance relative to the superconducting substrate [22]. However, they surprisingly have nearly identical gap size, despite their coherence peaks having slightly different intensity. BCS fitting to the spectra indicates superconducting gap sizes for the $0^{\circ}$ and $24.2^{\circ}$ domains are both $0.92 \mathrm{meV}$, which confirms the negligible difference between them.
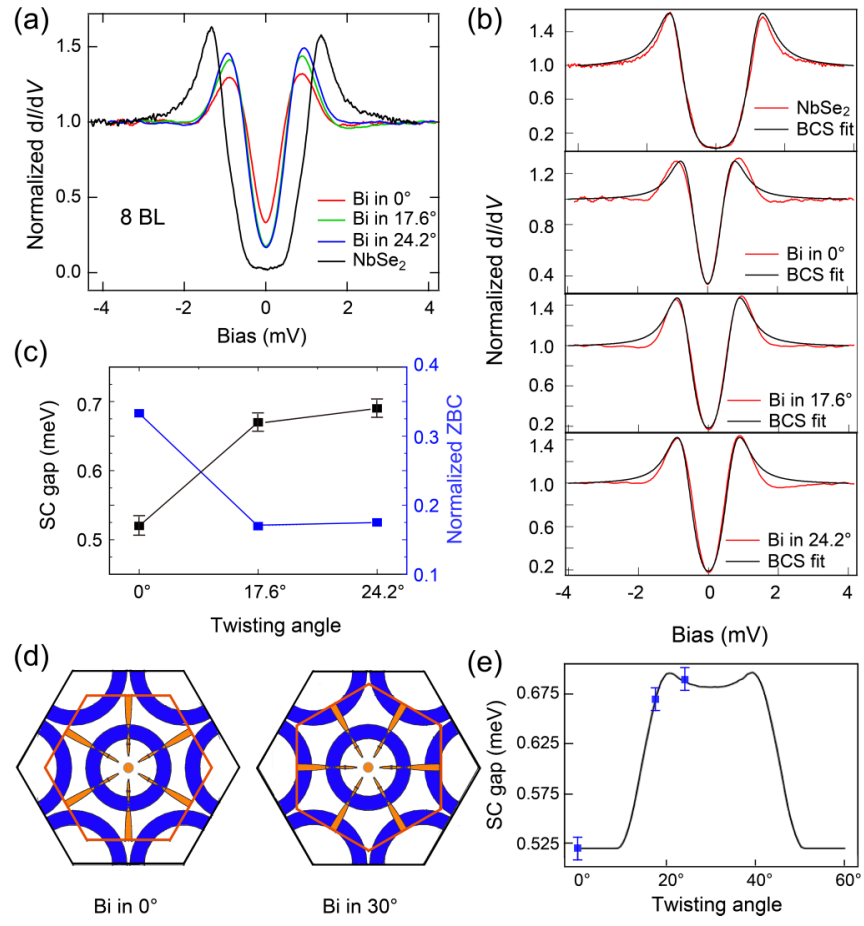

FIG. 2. Superconducting proximity gap of 8-BL Bi films with different twisting angles. (a) Normalized tunneling spectra of 8-BL Bi films, showing the typical twisting angle dependent superconducting proximity gap. The spectroscopic locations are marked with colored solid dots in Fig. 1(b). Spectroscopic conditions: $V_{\mathrm{b}}=4 \mathrm{mV}$, $I_{\mathrm{t}}=100 \mathrm{pA}, V_{\mathrm{mod}}=35 \mu \mathrm{V}$. (b) BCS fitting of the superconducting proximity gap in (a). (c) Fitted superconducting gap size and measured normalized ZBC of the spectra in (a). (d) Schematics showing the Fermi surfaces of 8-BL Bi film (orange color) and $\mathrm{NbSe}_{2}$ (blue color). The Brillouin zone boundary of $\mathrm{Bi}\left(\mathrm{NbSe}_{2}\right)$ is depicted with a red (black) hexagon. (e) Calculated superconducting (SC) proximity gap of Bi films (black curve) with different twisting angle relative to $\mathrm{NbSe}_{2}$. The blue dots represent the experimental values from (c).

To understand the contrast behavior of the superconducting proximity effect with the stacking angle and the film thickness, we scrutinize the electronic structure of the Bi films and the $\mathrm{NbSe}_{2}$ substrate. Previous studies with angle-resolved photoemission spectroscopy have reported the evolution of band structures of Bi films with thickness $[25,26]$, which corroborates our observed thickness dependent conductance peak associated with band van Hove singularity. Specifically, the Fermi surface of 8-BL Bi film has a hole pocket at the central Brillouin zone and six electron pockets at the $M$ points of the zone boundary [Fig. 2(d)]. With decreasing film thickness, the electron pockets shrink their contours, and become barely seen at thin film thickness of 4 BL [Fig. 4(d)], as a result of changing interlayer coupling $[25,26]$. On the other hand, the Fermi surface contour of $\mathrm{NbSe}_{2}$ also has valley character with a hole pocket at the $\Gamma$ point and six electron pockets at the $\mathrm{K}$ points of the surface Brillouin zone [27]. The relative stacking angle between $\mathrm{Bi}$ and $\mathrm{NbSe}_{2}$ crucially determines the valley dependent wave function coupling at the interface, which makes the superconducting proximity effect of Bi endowed with valley dependence. With decreasing film thickness, the electron pockets at the $M$ point are depleted, 

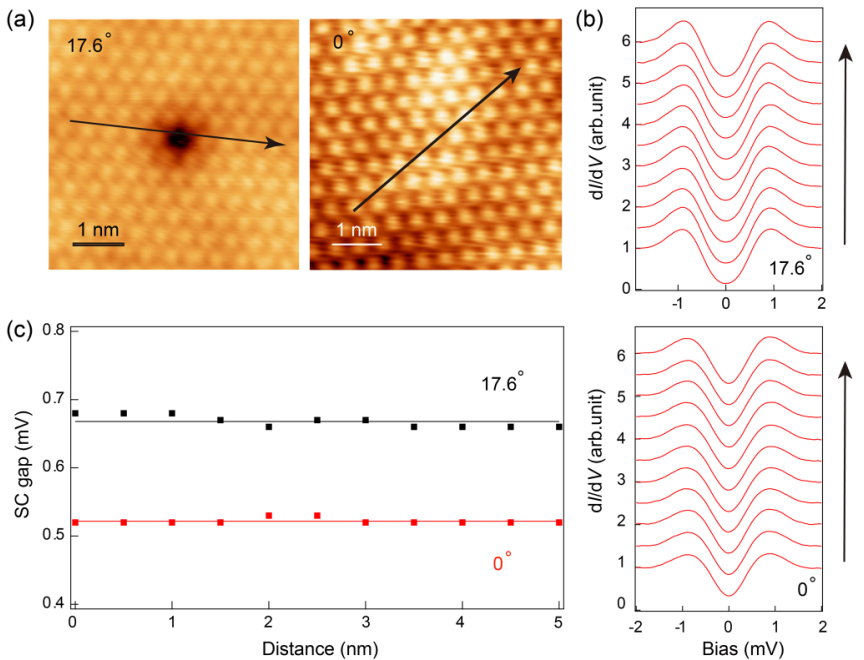

FIG. 3. Evaluation of the variation of the superconducting proximity gap at different locations. (a) STM images of two domains of $17.6^{\circ}$ and $0^{\circ}$. The imaging conditions are $200 \mathrm{mV}, 500 \mathrm{pA}$ and $400 \mathrm{mV}, 500 \mathrm{pA}$ for the $17.6^{\circ}$ and $0^{\circ}$ domains, respectively. There is a vacancy defect in the image of the $17.6^{\circ}$ domain. (b) Tunneling spectra of the superconducting proximity gap measured along the black lines in (a) with a modulation of $0.035 \mathrm{mV}$ (rms). (c) Superconducting proximity gap size fitted from the spectra in (b). The black and red lines mark the statistical average of the gap sizes of the $17.6^{\circ}$ and $0^{\circ}$ domains, respectively. (a)

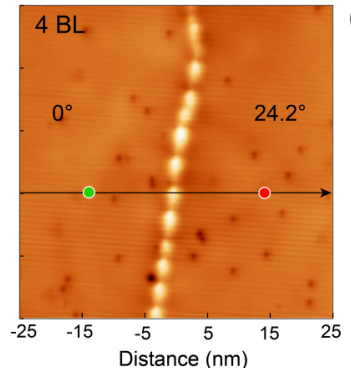

(c)

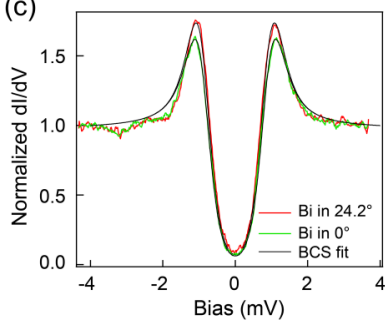

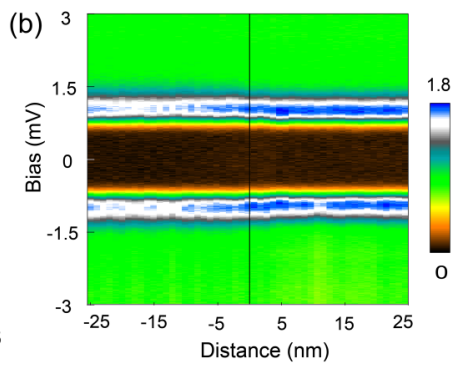

(d)

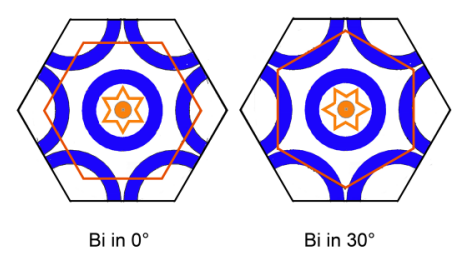

FIG. 4. Superconducting proximity gap of 4-BL Bi films with different twisting angles. (a) STM image $\left(V_{\mathrm{b}}=1 \mathrm{~V}, I_{\mathrm{t}}=10 \mathrm{pA}\right)$ of a 4-BL Bi film with a domain boundary in the middle. The twisting angles between $\mathrm{Bi}$ films and $\mathrm{NbSe}_{2}$ are indicated. (b) $2 \mathrm{D}$ conductance plot taken along the black line in (a), showing the evolution of the superconducting proximity gap across the domain boundary. The black line marks the location of the domain boundary. Spectroscopic conditions: $V_{\mathrm{b}}=4 \mathrm{mV}, I_{\mathrm{t}}=100 \mathrm{pA}, V_{\text {mod }}=35 \mu \mathrm{V}$. (c) Tunneling spectra taken at the marked spots in (a), showing typical superconducting proximity gaps of the two domains. (d) Schematics showing the Fermi surfaces of 4-BL Bi film (orange) and $\mathrm{NbSe}_{2}$ (blue). The Brillouin zone boundary of $\mathrm{Bi}\left(\mathrm{NbSe}_{2}\right)$ is depicted with a red (black) hexagon. losing the valley character of the bands and the concomitant influence on superconducting proximity effect.

To examine the above scenario, we use a standard microscopic model, accounting for the valley dependent coupling at the $\mathrm{Bi} / \mathrm{NbSe}_{2}$ interface, to evaluate the proximity-induced pairing potential in the $\mathrm{Bi}$ thin film (Appendix $\mathrm{A}$ ). Here we consider the $\mathrm{Bi} / \mathrm{NbSe}_{2}$ system as infinite in the twodimensional (2D) $x-y$ plane. At the $\mathrm{Bi} / \mathrm{NbSe}_{2}$ interface, the induced gap $\Delta_{s}$ in $\mathrm{Bi}$ mainly comes from the inter- and intrachannel couplings, whose amplitudes are referred to as $\lambda_{0}$ and $\lambda_{1}$, respectively. The induced gap $\Delta_{s}$ has the form $\Delta_{s}=\lambda_{0}+\lambda_{1}$ [28-30]. As the $x-y$ plane is infinitely large, the inter- and intrachannel couplings correspond to the scattering processes which violate and conserve the in-plane momentum, respectively. In this sense, the interchannel coupling $\lambda_{0}$, which normally comes from the roughness of the interface, is independent of the twisting angle $\phi$ between $\mathrm{Bi}$ and $\mathrm{NbSe}_{2}$ The intrachannel coupling $\lambda_{1}$ is proportional to the amplitude of the wave function of $\mathrm{Bi}$ at the interface and the local density of states of $\mathrm{NbSe}_{2}$ at the Fermi level, and thus takes the form $\lambda_{1}=k S(\phi)$, with $S(\phi)$ the Fermi surface overlap between the $\mathrm{Bi}$ and $\mathrm{NbSe}_{2}$ [Fig. 2(d)] and $k$ is the proportional coefficient, reflecting the amplitude of the angle dependent component of the gap [28-30]. Therefore, the angle dependent proximity gap $\Delta_{s}$ in Bi can be written as $\Delta_{s}=\lambda_{0}+k S(\phi)$ [28-30]. As a result, we can obtain $\Delta_{s}$ as a function of $\phi$, with only two fitting parameters, $\lambda_{0}=0.52 \mathrm{meV}$ and $k=24.55 \mathrm{meV} \AA^{2}$ for the case of $8-\mathrm{BL}$ Bi film. The results given in Fig. 2(e) have nice agreement with the experimentally measured proximity gap sizes. At small twisting angle $\phi, \Delta_{s}$ does not change because there is no overlap between the Fermi surfaces of $\mathrm{Bi}$ and $\mathrm{NbSe}_{2}$. As $\phi$ increases, the electron pockets of $\mathrm{Bi}$ at the $M$ points and the electron pockets of $\mathrm{NbSe}_{2}$ at the $K$ points have finite overlap, so that $\Delta_{s}$ becomes angle dependent with the periodicity of $60^{\circ}$. For the 4-BL Bi film, its electron pockets at the $M$ points are negligible Fig. 4(d), so $\Delta_{s}$ is independent of $\phi$.

We have also studied the lateral proximity effect between the $0^{\circ}$ and $17.6^{\circ}$ domains on the $8-\mathrm{BL}$ Bi. Figures 5 (a) and 5(b) show consecutive spectra taken perpendicular to the domain boundary. Notably, the superconducting gap at the $17.6^{\circ}$ domain stays constant, and gradually shrinks to that of the interior $0^{\circ}$ domain when passing through the domain boundary. This demonstrates the superconducting gap variation happens at the side of the superconductor with smaller gap size. This observation is further substantiated by the normalized ZBC mapping [Fig. 5(c)]. The normalized ZBC is uniformly small with a value of $0.1 \pm ; 0.01$ on the $17.6^{\circ}$ domain, and monotonically increases away from the boundary towards the interior domain. Such behavior conforms to the framework of the Usadel theory [31], which depicts the penetration of Cooper pairs through the $\mathrm{S} 1 / \mathrm{S} 2$ interface, where $\mathrm{S} 1$ has a larger superconducting gap, in a diffusive regime [32]; i.e., the superconducting coherence length of the S2 superconductor $\mathcal{E}_{2}$ is smaller than its mean free path $l_{e 2}$. For a quantitative analysis, we take the normalized ZBC from the averaged conductance map in Fig. 5(c) and get their evolution as a function of distance [Fig. 5(d)].

According to the Usadel equation (Appendix B), both the superconducting proximity gap size $\Delta_{2}$ and the associated 

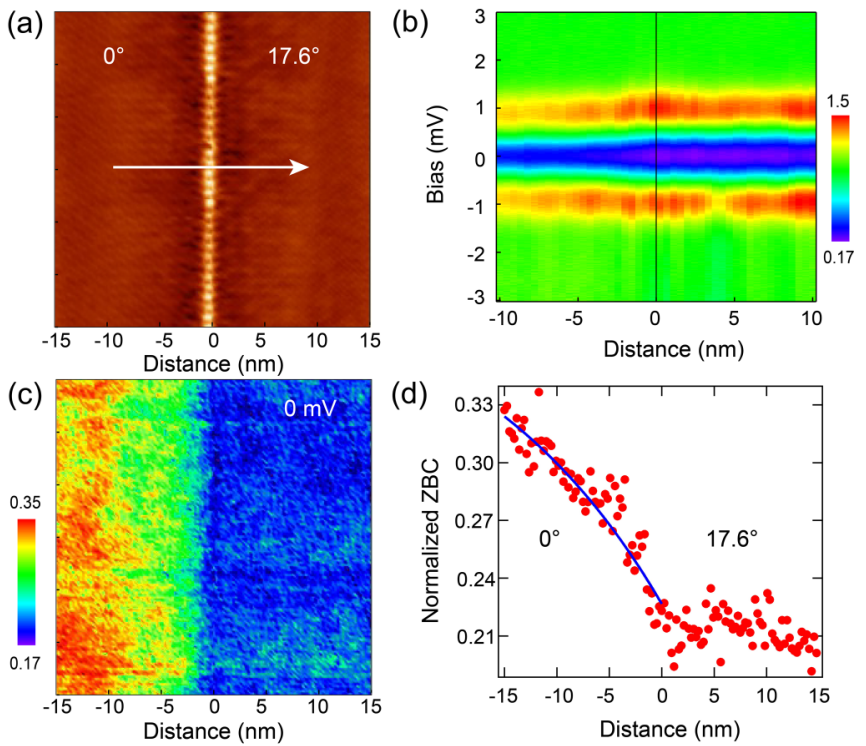

(e)

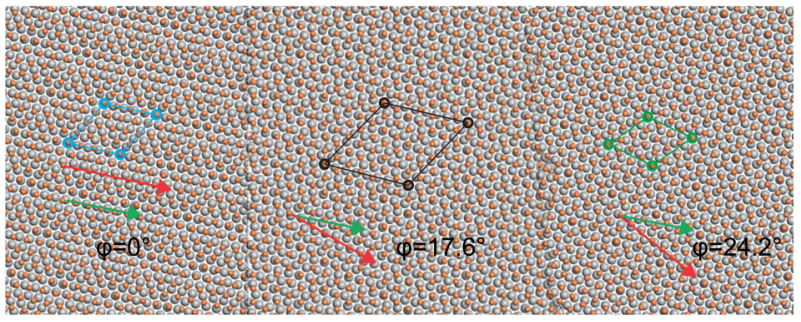

FIG. 5. Lateral superconducting proximity effect between two domains of 8-BL Bi films. (a) STM image $\left(V_{\mathrm{b}}=300 \mathrm{mV}, I_{\mathrm{t}}=\right.$ $500 \mathrm{pA}$ ) of 8 -BL Bi films with a domain boundary in the middle. The twisting angles between $\mathrm{Bi}$ and $\mathrm{NbSe}_{2}$ are indicated. (b) 2D conductance plot measured along the white line in (a). The black line marks the location of the domain boundary. Spectroscopic conditions: $V_{\mathrm{b}}=3 \mathrm{mV}, I_{\mathrm{t}}=100 \mathrm{pA}, V_{\bmod }=35 \mu \mathrm{V}$. (c) Tunneling conductance mapping at the imaged area in (a) taken at zero bias. Spectroscopic conditions: $I_{\mathrm{t}}=100 \mathrm{pA}, V_{\text {mod }}=35 \mu \mathrm{V}$. (d) Normalized $\mathrm{ZBC}$ as a function of distance averaged from the mapping in (c). The solid blue line is an Usadel fitting to the normalized ZBC data. (e) Moiré pattern of different domains. The top layer Se atoms of the $\mathrm{NbSe}_{2}$ substrate and the $\mathrm{Bi}$ atoms are represented with gray and orange balls, respectively. The relative orientations of the two constituent layers are indicated with green and red arrows for the Se lattice and Bi lattice, respectively. The moiré units for each domain are marked.

normalized ZBC follow a relation with distance $x$ from the interface, $\Delta_{2} \propto e^{-x / \mathcal{E}_{2}}$, and the superconducting gap at the larger gap side is barely affected. An Usadel fitting to Fig. 5(d) delivers a $\mathcal{E}_{2}$ of $\sim 18 \mathrm{~nm}$. The diffusion constant $D_{2}$ of the S2 superconductor can be obtained as $4.7 \mathrm{~cm}^{2} / \mathrm{s}$ from the relation $\mathcal{E}_{2}=\sqrt{\hbar D_{2} / \Delta_{1}}$ given by the Usadel theory [33,34]. This diffusion length is orders of magnitude smaller than the bulk value [35]. Since the diffusion constant $D=v_{F} l_{e} / 2$, there are two possible sources of the decreased diffusion constant, namely, the reduction of Fermi velocity $v_{F}$ and/or the mean free path $l_{e}$. Since $v_{F}$ of 8-BL film is close to the bulk value, $l_{e}$ should get significantly smaller. As is shown from the STM topography, our film is of high quality with a negligible number of defects, excluding the possibility of defect-induced reduction of $l_{e}$. Rather, the thin film is subject to interface scattering [36], presumably from the moiré pattern at the $\mathrm{Bi} / \mathrm{NbSe}_{2}$ interface.

There also exists an inverse proximity effect between the two domains. Notably, normalized ZBC of the $17.6^{\circ}$ domain gradually decreases from $\sim 0.22$ at the boundary [Fig. 5(d)] to $\sim 0.17$ in the bulk [Fig. 2(c)], where an exponential decay should be expected from the Usadel equation [34,37]. However, it is difficult to obtain a reliable fitting of the inverse proximity effect in the $17.6^{\circ}$ domain due to the small decay values and the relatively large error bars [Fig. 5(d)]. Nevertheless, the decay length can still be estimated from the moiré density of the domains, which is the ascribed dominant scattering source of the current system in the dirty limit. The moire density in the $17.6^{\circ}$ domain is $\sim 1 / 4$ that of the $0^{\circ}$ domain [Fig. 5(e)]. This renders its mean free path four times larger, resulting in its decay length being at least twice as large. This is qualitatively consistent with the observed less obvious inverse proximity effect.

\section{CONCLUSION}

In conclusion, we have investigated the superconducting proximity effect of Bi films grown on a $\mathrm{NbSe}_{2}$ substrate, whose proximity gap exhibits prominent dependence on the interfacial stacking angle. Our theoretical modeling reveals its origin as the valley dependent Andreev reflection. Furthermore, the lateral proximity effect between the two domains of Bi films are observed, allowing quantitative determination of the mean free path of carriers. The valley dependent superconducting proximity effect here enriches the tunability of physical properties with the valley degree of freedom. This may enable application in constructing higher-order topological superconductivity [38], and building quantum devices based on tunable superconducting proximity coupling in valleytronic heterostructures [39].

\section{ACKNOWLEDGMENTS}

We thank Z. W. Zhu, J. H. Gao, and J. T. Lü for discussions. This work is funded by the National Key Research and Development Program of China (Grants No. 2017YFA0403501, No. 2016YFA0401003, and No. 2018YFA0307000), the National Science Foundation of China (Grants No. 11874161, No. 11774105, and No. 11674114).

\section{APPENDIX A: MODEL FOR VALLEY DEPENDENT SUPERCONDUCTING PROXIMITY EFFECT}

In this section, we give the details of the derivation for the relation $\Delta_{\mathrm{s}}=\lambda_{0}+k S(\phi)$. The contribution of the $\mathrm{NbSe}_{2}$ can be regarded as the interface self-energy of the Bi films which is given by $\Sigma(k, \omega)=-\left(\lambda_{0}+\lambda_{1}\right) \frac{\left(\omega \tau_{0}+\Delta \tau_{x}\right)}{\sqrt{\Delta^{2}-\omega^{2}}}$ [28-30]. Here, $\lambda_{0}, \lambda_{1}$ are the inter- and intrachannel couplings couplings, respectively, $\Delta$ is the superconducting gap of $\mathrm{NbSe}_{2} ; \tau$ is the Pauli matrix acting in the particle-hole space. As the detected proximity-induced gap is less than $2 / 3$ of the $\mathrm{NbSe}_{2}$ gap, we neglect the dynamic effect and take $\omega=0$, so the induced gap $\Delta_{s}$ in Bi has the simple form $\lambda_{0}+\lambda_{1}$. The intrachannel coupling $\lambda_{1}$ has the form $\lambda_{1}=t^{2} \int\left|\Psi\left(k_{\|}\right)\right|^{2} v\left(\epsilon_{F}, k_{\|}\right) d^{2} k_{\|}$, with $t$ the transparency of the interface, $\Psi\left(k_{\|}\right)$the amplitude of 

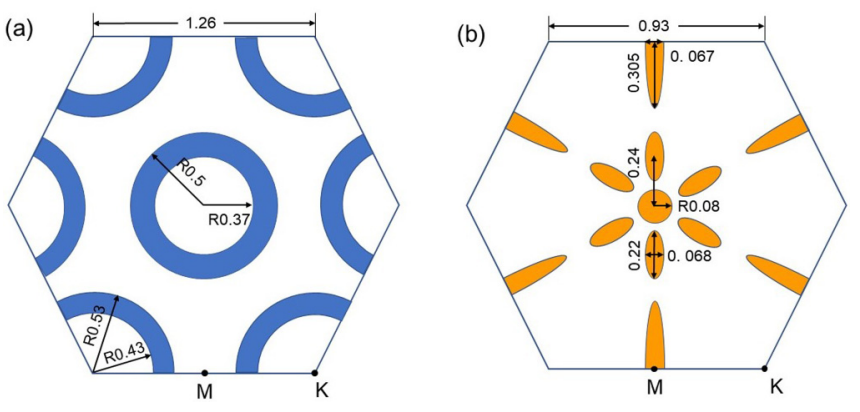

FIG. 6. The parameters of the two-dimensional Fermi surface in $\mathrm{NbSe}_{2}$ (a) and $\mathrm{Bi}(\mathrm{b})$.

the wave function of $\mathrm{Bi}$ at the interface with given in-plane momentum $k_{\|}$, and $v\left(\epsilon_{F}, k_{\|}\right)$the local density of states of $\mathrm{NbSe}_{2}$ at the Fermi level $\epsilon_{F}$ [28-30]. Therefore $\lambda_{1}$ is related to both the amplitude of the wave function of $\mathrm{Bi}$ and the local density of states of $\mathrm{NbSe}_{2}$ at Fermi level. Moreover, because of the in-plane momentum conservation for the intrachannel coupling, $\lambda_{1}$ is linearly proportional to the overlap of the Fermi surfaces $S(\phi)$ between $\mathrm{Bi}$ and $\mathrm{NbSe}_{2}$. Finally, we obtain the relation $\Delta_{\mathrm{s}}=\lambda_{0}+k S(\phi)$, as given in the main text. The parameters of the two-dimensional Fermi surface of Bi and $\mathrm{NbSe}_{2}$ are extracted from the experiments $[26,27]$ which are given in Fig. 6. Note that in Ref. [26], the Fermi surfaces of 6- and 10-BL Bi are nearly identical, while the 8-BL Fermi surface is lacking. We therefore take the Fermi surface of $10 \mathrm{BL}$ from the reference in our modeling with both the confined bulk states (around the $\bar{\Gamma}$ point) and the surface states (around the $\bar{M}$ point) considered.

\section{APPENDIX B: USADEL SOLUTION FOR LATERAL SUPERCONDUCTING PROXIMITY EFFECT}

It is natural to assume that there is no phase difference between the different domains. Thus, we can start from the $\theta$ parametrized Usadel equation at $E=0$ as in Ref. [32].

$$
\frac{\hbar D}{2} \frac{\partial^{2} \vartheta(x)}{\partial x^{2}}-i \Delta \cosh \vartheta(x)=0,
$$

where $\Delta$ and $D$ are the gap function and diffusive constant for the domain with twisted angle $17.6^{\circ}$. Here we assume the gap function has a sudden change across the domain interface but remains constant in each domain. Similar to Ref. [32], we replace the variable $\vartheta$ by $\theta$ through the relation $\vartheta(x)=$ $\theta(x)+i \frac{\pi}{2}$ so that the equation becomes

$$
\frac{\hbar D}{2} \frac{\partial^{2} \theta(x)}{\partial x^{2}}-\Delta \sinh \theta(x) \approx \frac{\hbar D}{2} \frac{\partial^{2} \theta(x)}{\partial x^{2}}-\Delta \theta(x)=0 .
$$

Here we make the approximation that $\sinh \theta \approx \theta$ for small $\theta$. Then, the above equation gives us the solution $\theta(x)=$ $-\mathrm{i} A \exp \left(-\frac{x}{\xi}\right)$ with $\xi=\sqrt{\frac{\hbar D}{2 \Delta}}$, and $|A| \ll 1$ is some constant. The density of states at $E=0$ can be obtained as

$$
\operatorname{LDOS}(x, E=0)=N_{0} \operatorname{Re}[\cosh \vartheta] \approx N_{0}|A| e^{-\frac{x}{\xi}},
$$

where $N_{0}$ is the normal density of states. As we know that the density of states in the superconducting regime is much smaller than $N_{0}$, so we have $|A| \ll 1$ which is consistent with our previous assumption. As the zero bias conductance is proportional to the LDOS, we thus use $\mathrm{Ce}^{-\frac{x}{\xi}}+C_{0}$ to fit our data. Here considering the off-site conductance, we add $\mathrm{C}_{0}$ in the fit equation.
[1] Z. Zhu, B. Fauqué, K. Behnia, and Y. Fuseya, Magnetoresistance and valley degree of freedom in bulk bismuth, J. Phys.: Condens. Matter 30, 313001 (2018).

[2] Z. Zhu, J. Wang, H. Zuo, B. Fauqué, R. D. McDonald, Y. Fuseya, and K. Behnia, Emptying Dirac valleys in bismuth using high magnetic fields, Nat. Commun. 8, 15297 (2017).

[3] K. Takashina, Y. Ono, A. Fujiwara, Y. Takahashi, and Y. Hirayama, Valley Polarization in $\mathrm{Si}(100)$ at Zero Magnetic Field, Phys. Rev. Lett. 96, 236801 (2006).

[4] F. Bussolotti et al., Roadmap on finding chiral valleys: Screening 2D materials for valleytronics, Nano Future 2, 032001 (2018).

[5] J.-T. Sun and S. Meng, The valley degree of freedom of an electron, Acta. Phys. Sin. 64, 187301 (2015).

[6] D. Xiao, M. C. Chang, and Q. Niu, Berry phase effects on electronic properties, Rev. Mod. Phys. 82, 1959 (2010).

[7] K. F. Mak, K. L. McGill, J. Park, and P. L. McEuen, The valley Hall effect in $\mathrm{MoS}_{2}$ transistors, Science 344, 1489 (2014).
[8] K. F. Mak, K. He, J. Shan, and T. F. Heinz, Control of valley polarization in monolayer $\mathrm{MoS}_{2}$ by optical helicity, Nat. Nanotechnol. 7, 494 (2012).

[9] H. Zeng, J. Dai, W. Yao, D. Xiao, and X. Cui, Valley polarization in $\mathrm{MoS}_{2}$ monolayers by optical pumping, Nat. Nanotechnol. 7, 490 (2012).

[10] J. M. Lu, O. Zeliuk, I. Leermakers, N. F. Q. Yuan, U. Zeitler, K. T. Law, and J. T. Ye, Evidence for two-dimensional Ising superconductivity in gated $\mathrm{MoS}_{2}$, Science 350, 1353 (2015).

[11] P. G. De Gennes, Boundary effects in superconductors, Rev. Mod. Phys. 36, 225 (1964).

[12] A. I. Buzdin, Proximity effects in superconductorferromagnet heterostructures, Rev. Mod. Phys. 77, 935 (2005).

[13] V. Mourik, K. Zuo, S. M. Frolov, S. R. Plissard, E. P. A. M. Bakkers, and L. P. Kouwenhoven, Signatures of Majorana fermions in hybrid superconductor-semiconductor nanowire devices, Science 336, 1003 (2012). 
[14] S. Nadj-Perge, I. K. Drozdov, J. Li, H. Chen, S. Jeon, J. Seo, A. H. MacDonald, B. A. Bernevig, and A. Yazdani, Observation of Majorana fermions in ferromagnetic atomic chains on a superconductor, Science 346, 602 (2014).

[15] J.-P. Xu, C. Liu, M.-X. Wang, J. Ge, Z.-L. Liu, X. Yang, Y. Chen, Y. Liu, Z.-A. Xu, and C.-L. Gao et al., Artificial Topological Superconductor by the Proximity Effect, Phys. Rev. Lett. 112, 217001 (2014).

[16] P. Zhang, K. Yaji, T. Hashimoto, Y. Ota, T. Kondo, K. Okazaki, Z. Wang, J. Wen, G. D. Gu, and H. Ding et al., Observation of topological superconductivity on the surface of an iron-based superconductor, Science 360, 182 (2018).

[17] A. Allain, Z. Han, and V. Bouchiat, Electrical control of the superconducting-to-insulating transition in graphene-metal hybrids, Nat. Mater. 11, 590 (2012).

[18] V. E. Calado, S. Goswami, G. Nanda, M. Diez, A. R. Akhmerov, K. Watanabe, T. Taniguchi, T. M. Klapwijk, and L. M. K. Vandersypen, Ballistic Josephson junctions in edge-contacted graphene, Nat. Nanotechnol. 10, 761 (2015).

[19] S. Takei, B. M. Fregoso, H.-Y. Hui, A. M. Lobos, and S. Das Sarma, Soft Superconducting Gap in Semiconductor Majorana Nanowires, Phys. Rev. Lett. 110, 186803 (2013).

[20] Ö. Gül, H. Zhang, F. K. de Vries, J. van Veen, K. Zuo, V. Mourik, S. Conesa-Boj, M. P. Nowak, D. J. van Woerkom, and M. Quintero-Pérez et al., Hard superconducting gap in InSb nanowires, Nano Lett. 17, 2690 (2017).

[21] L. Peng, J.-J. Xian, P. Tang, A. Rubio, S.-C. Zhang, W. Zhang, and Y.-S. Fu, Visualizing topological edge states of single and double bilayer $\mathrm{Bi}$ supported on multibilayer $\mathrm{Bi}(111)$ films, Phys. Rev. B 98, 245108 (2018).

[22] H.-H. Sun, M.-X. Wang, F. Zhu, G.-Y. Wang, H.-Y. Ma, Z.-A. $\mathrm{Xu}$, Q. Liao, Y. Lu, C.-L. Gao, and Y.-Y. Li et al., Coexistence of topological edge state and superconductivity in bismuth ultrathin film, Nano Lett. 17, 3035 (2017).

[23] L. Peng, J. Qiao, J.-J. Xian, Y. Pan, W. Ji, W. H. Zhang, and Y.-S. Fu, Unusual electronic states and superconducting proximity effect of Bi films modulated by a $\mathrm{NbSe}_{2}$ substrate, ACS Nano 13, 1885 (2019).

[24] Y. L. Huang, Z. Ding, W. Zhang, Y.-H. Chang, Y. Shi, L.-J. Li, Z. Song, Y. J. Zheng, D. Chi, S. Y. Quek, and A. T. S. Wee, Gap states at low-angle grain boundaries in monolayer tungsten diselenide, Nano Lett. 16, 3682 (2016).

[25] L. Miao, M.-Y. Yao, W. Ming, F. Zhu, C. Q. Han, Z. F. Wang, D. D. Guan, C. L. Gao, C. Liu, F. Liu, D. Qian, and J.-F. Jia, Evolution of the electronic structure in ultrathin Bi(111) films, Phys. Rev. B 91, 205414 (2015).

[26] T. Hirahara, T. Shirai, T. Hajiri, M. Matsunami, K. Tanaka, S. Kimura, S. Hasegawa, and K. Kobayashi, Role of Quantum and Surface-State Effects in the Bulk Fermi-Level Position of Ultrathin Bi Films, Phys. Rev. Lett. 115, 106803 (2015).
[27] F. Flicker and J. van Wezel, Charge order from orbitaldependent coupling evidenced by $\mathrm{NbSe}_{2}$, Nat. Commun. 6, 7034 (2015).

[28] T. D. Stanescu, J. D. Sau, R. M. Lutchyn, and S. Das Sarma, Proximity effect at the superconductor-topological insulator interface, Phys. Rev. B 81, 241310 (2010).

[29] J. D. Sau, S. Tewari, R. M. Lutchyn, T. D. Stanescu, and S. Das Sarma, Non-Abelian quantum order in spin-orbitcoupled semiconductors: Search for topological majorana particles in solid-state systems, Phys. Rev. B 82, 214509 (2010).

[30] T. D. Stanescu, R. M. Lutchyn, and S. Das Sarma, Majorana fermions in semiconductor nanowires, Phys. Rev. B 84, 144522 (2011).

[31] K. D. Usadel, Generalized Diffusion Equation for Superconducting Alloys, Phys. Rev. Lett. 25, 507 (1970).

[32] V. Cherkez, J. C. Cuevas, C. Brun, T. Cren, G. Ménard, F. Debontridder, V. S. Stolyarov, and D. Roditchev, Proximity Effect between Two Superconductors Spatially Resolved by Scanning Tunneling Spectroscopy, Phys. Rev. X 4, 011033 (2014).

[33] L. Serrier-Garcia, J. C. Cuevas, T. Cren, C. Brun, V. Cherkez, F. Debontridder, D. Fokin, F. S. Bergeret, and D. Roditchev, Scanning Tunneling Spectroscopy Study of the Proximity Effect in a Disordered Two-Dimensional Metal, Phys. Rev. Lett. 110, 157003 (2013).

[34] C. Brun, T. Cren, V. Cherkez, F. Debontridder, S. Pons, D. Fokin, M. C. Tringides, S. Bozhko, L. B. Ioffe, and B. L. Altshuler et al., Remarkable effects of disorder on superconductivity of single atomic layers of lead on silicon, Nat. Phys. 10, 444 (2014).

[35] A. Collaudin, B. Fauqué, Y. Fuseya, W. Kang, and K. Behnia, Angle Dependence of the Orbital Magnetoresistance in Bismuth, Phys. Rev. X 5, 021022 (2015).

[36] A. Stępniak, M. Caminale, A. A. Leon Vanegas, H. Oka, D. Sander, and J. Kirschner, Temperature dependence of the superconducting proximity effect quantified by scanning tunneling spectroscopy, AIP Adv. 5, 017125 (2015).

[37] H. le Sueur, P. Joyez, H. Pothier, C. Urbina, and D. Esteve, Phase Controlled Superconducting Proximity Effect Probed by Tunneling Spectroscopy, Phys. Rev. Lett. 100, 197002 (2008).

[38] R. X. Zhang, W. S. Cole, X. Wu, and S. Das Sarma, HigherOrder Topology and Nodal Topological Superconductivity in $\mathrm{Fe}(\mathrm{Se}, \mathrm{Te})$ Heterostructures, Phys. Rev. Lett. 123, 167001 (2019).

[39] M. Kim, G. H. Park, J. Lee, J. H. Lee, J. Park, H. Lee, G. H. Lee, and H. J. Lee, Strong proximity Josephson coupling in vertically stacked $\mathrm{NbSe}_{2}$-graphene-NbSe$e_{2}$ van der Waals junctions, Nano Lett. 17, 6125 (2017). 